17.1

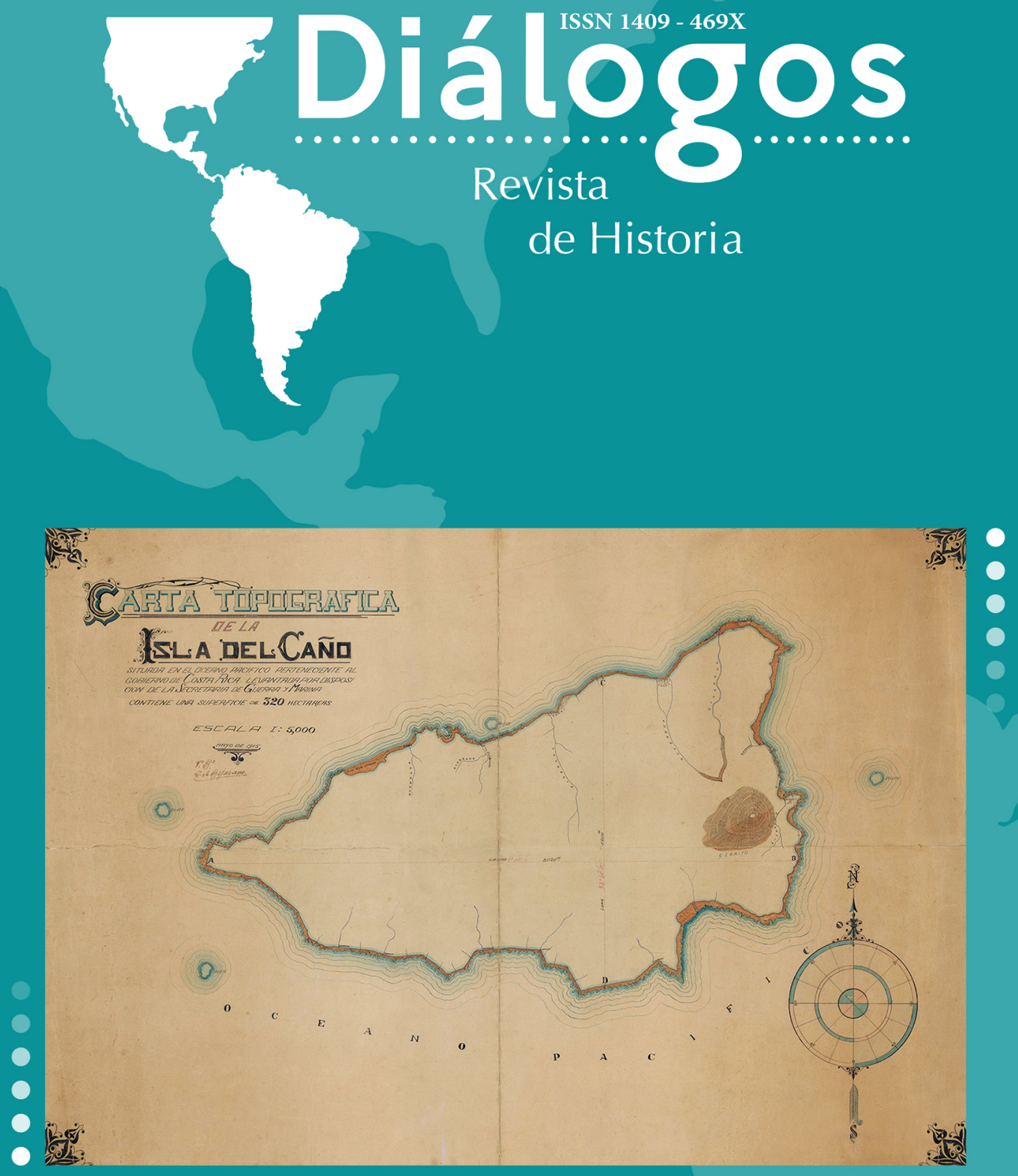

Escuela de Historia. Universidad de Costa Rica

Enero-junio 2016

url: http://revistas.ucr.ac.cr/index.php/dialogos/index 


\title{
EN LOS ORÍGENES DE LA PROFESIÓN: LA INSTITUCIONALIZACIÓN DE LA FORMACIÓN PROFESIONAL DEL TRABAJO SOCIAL COSTARRICENSE (1942-1952)
}

Milton Ariel Brenes Rodríguez

\begin{abstract}
Resumen
En este artículo se reconstruye el proceso de institucionalización de la formación profesional del Trabajo Social costarricense, durante el periodo de 1942 a 1952. Para tales efectos, considerando la base contextual que permite dicha profesionalización, se retoman aspectos relacionados con las tendencias partidarias, personal docente, oscilaciones administrativas, planes de estudio y fundamentaciones de la formación en la Escuela de Trabajo Social, tanto en su constitución, su carácter semiprivado, así como su entrada a la Universidad de Costa Rica.
\end{abstract}

Palabras claves: estado, política social, cuestión social, Escuela de Trabajo Social.

\section{ON THE ORIGINS OF THE PROFESSION: THE INSTITUTIONALIZATION OF TRAINING OF COSTA RICAN SOCIAL WORK (1942-1952)}

\begin{abstract}
In this article, the author reconstructs and describes the process of institutionalizing of training of Costa Rican Social Work profession during the historical period of 1942 to 1952 . For this purpose, considering the contextual basis that allowed such professionalization, various aspects are taken up in the analysis, such as party trends, faculty, administrative oscillations, curriculum and foundations of the School of Social Work, including in its constitution, semi-private, and its entry to the University of Costa Rica.
\end{abstract}

Keywords: state, social policy, social question, School of Social Work.

\footnotetext{
- Milton Ariel Brenes Rodríguez • Costarricense. Licenciado en Trabajo Social, Universidad - de Costa Rica. Docente en la Escuela de Trabajo Social, Universidad de Costa Rica.

- Contacto correo electrónico: arielbr227@hotmail.com
} 


\title{
BREVES PREMISAS TEÓRICAS E HISTÓRICAS A MODO DE INTRODUCCIÓN
}

En febrero de 1942, Héctor Beeche Luján, un abogado cercano a los sectores políticos costarricenses, le planteó al secretario de Educación Pública Luis Demetrio Tinoco:

\begin{abstract}
El actual gobierno, presidido por un mandatario hondamente preocupado por todas las cuestiones sociales [Rafael Ángel Calderón Guardia], acaba de aprobar un Código Penal que prevee[sic] una nueva organización en materia penitenciaria; asímismo[sic] ha logrado la fundación de una Caja de Seguros Sociales, que es el paso tal vez más avanzado que se haya dado en toda Centro América en el campo de la Previsión Social. Entre toda esa legislación social, y a pesar de tan excelentes instituciones sociales, se nota únicamente una laguna: la ausencia de personal técnico, debidamente preparado para actuar en el campo de la acción social. (Archivo Nacional de Costa Rica, 1942, Signatura 4722. Folio 66).
\end{abstract}

Las intenciones de Beeche Luján en esta misiva eran claras, iniciaba las gestiones para formalizar la fundación de la primera Escuela de Servicio Social en Costa Rica. ${ }^{1}$ Con el propósito de reflexionar sobre ese proceso, el presente artículo abordará en términos generales la institucionalización de la formación profesional del Trabajo Social costarricense durante el periodo de 1942 a 1952.

En ese sentido, es necesario iniciar planteando algunas breves premisas teóricas que posibilitan la explicación de la génesis y reproducción del Trabajo Social como profesión.

De acuerdo con ello, cabe señalar que las gestiones de Beeche Luján reflejan un aspecto inherente al proceso de constitución profesional que resulta importante considerar: las condiciones históricas de las cuales deviene la legitimidad del Trabajo Social y por ende su institucionalización, se encontraba en ascenso.

Con la intención de ampliar tales planteamientos, se debe indicar que el surgimiento de la profesión no se explica de manera endógena, sino que las condiciones del proyecto reformista costarricense, para la fecha en ascenso, exigían profesiones como el Trabajo Social que intervinieran a través de la naciente política social en la llamada cuestión social.

De esta forma, recurriendo a la teoría social crítica que estudia el origen de la profesión, es válido afirmar que el surgimiento del Trabajo Social se presenta cuando existe una mediación política entre la cuestión social y el Estado; mediación que alcanza un nivel de instrumentalización a través de las políticas sociales, cuyo ejecutor en buena parte es el Trabajo Social (Montaño, 2000).

A partir de estas premisas, el surgimiento de la profesión no se debe reducir a las gestiones de Beeche Luján por fundar una Escuela de Servicio Social, ni tampoco a la Escuela misma, sino que su comprensión se encuentra en las condiciones históricas (económicas, políticas, sociales y culturales) que posibilitaron su constitución, y generaron una ruptura con sus anteriores protoformas (Netto, 1992). 


\section{LAS OSCILACIONES ADMINISTRATIVAS Y CURRICULARES DE LA ESCUELA DE SERVICIO SOCIAL}

La solicitud de Beeche Luján por fundar una Escuela de Servicio Social fue acogida por el Ministerio de Salud Pública, la Caja Costarricense de Seguro Social (CCSS) y el Patronato Nacional de la Infancia (PANI). En cuanto a ello, el beneplácito institucional por la Escuela refleja el trasfondo estructural, anteriormente mencionado, que reviste de legitimidad a la profesión (Archivo Nacional de Costa Rica, 1942, Signatura 4722. Folio 60-78).

Al respecto, cabe señalar que las fuentes que legitiman al Trabajo Social no emergen de una determinada connotación técnico-específica, sino que por el contrario, es el requerimiento estatal de profesiones que intervengan en el cuadrante del conflicto social el que exige y crea las condiciones para el desarrollo profesional (Montaño, 2000).

En relación con lo planteado, son elocuentes las palabras de Guillermo Padilla Castro, subgerente de la CCSS para el periodo en estudio. El señor Padilla afirmaba "es más: creo que la Escuela, una vez en funcionamiento, no sólo llenará un vacío en nuestro medio, sino que impulsará mucho los fines de educación que persigue la 'Caja”" (Archivo Nacional de Costa Rica, 1942, Signatura 4722. Folio 78). De igual manera, el Presidente del PANI aseguraba que la iniciativa resultaba "altamente beneficiosa para Costa Rica" (Archivo Nacional de Costa Rica, 1942, Signatura 4722. Folio 77).

De esta forma, con el apoyo institucional, desde marzo de 1942 la Escuela de Servicio Social se organizó de manera independiente con un carácter semiprivado (Archivo Universitario Rafael Obregón Loría, 1954). El proyecto en un inicio es planteado de esa forma porque a pesar de que desde febrero de 1942 Beeche Luján intentó incorporarla a la Universidad de Costa Rica, las gestiones para hacerlo tardaron dos años; por lo que durante este periodo en que no estaba vinculada a la Universidad, la Escuela fue financiada en mayor parte por las instituciones que habían acogido su apertura, es decir, el Ministerio de Salubridad, la CCSS y el PANI, así como por las mensualidades del estudiantado (Angulo, 2014; Fallas, 2010).

Los aspectos en mención permiten hacer énfasis en cómo la legitimidad profesional surge de esa función política que deviene de la institucionalidad, la cual está estrechamente relacionada con un determinado proyecto político, antes que con una delimitada estructura científica y método propio. Es decir, la profesión encuentra sus referentes constitutivos en la atención de las demandas sociales (Montaño, 2000).

Al ampliar en ese aspecto, se denota como el apoyo para la fundación de la Escuela fue acogido por la Universidad de Costa Rica, pero en este centro de estudios privó una relación subalterna y precarizada (Fallas, 2010). Al respecto, el argumento relacionado con una mayor preponderancia institucional en detrimento del sistema 
de saberes que subyace en la profesión, es una vía, no única, para comprender las oscilaciones que atravesó la Escuela de Servicio Social cuando se vinculó a la Universidad de Costa Rica.

El proceso para incorporarse a la Universidad, tal como se mencionó, inició con la solicitud planteada por Beeche Luján al secretario de Educación, Luis Demetrio Tinoco, en 1942. Dicha solicitud fue discutida en el Consejo Universitario el 10 de marzo del año en mención (Consejo Universitario de la Universidad de Costa Rica, 1942), desde entonces las autoridades universitarias crearon una comisión que valoró la pertinencia de la propuesta, la cual fue aprobada el 7 de marzo de 1944 (Consejo Universitario de la Universidad de Costa Rica, 1944).

Para la llegada de la Escuela de Servicio Social a la Universidad de Costa Rica, esta institución se encontraba permeada por los intereses de diferentes fracciones de los sectores hegemónicos. Inicialmente, por intereses vinculados con el calderonismo y el socialcristianismo, pero a su vez, los grupos relacionados con la socialdemocracia empezaron a pujar por un espacio en la institucionalidad y por ende en la conducción de la Universidad de Costa Rica. Dicha intención se consolidó posteriormente al conflicto armado de 1948, en donde la socialdemocracia y la intelectualidad asociada a ella afianzaron, no sin tensiones, su proyecto político en la institucionalidad costarricense (Fumero, 1997).

Con este panorama, resulta oportuno profundizar el mencionado proceso de marginal vinculación entre la Escuela de Servicio Social y la Universidad de Costa Rica. Al respecto, desde marzo de 1944 y hasta marzo de 1947, la Escuela fue una instancia anexa a la Facultad de Ciencias Económicas y Sociales con la cual no compartía espacio físico. Incluso en ocasiones se le instó a utilizar los salones de clases de las escuelas primarias públicas ante la negativa de la Escuela de Derecho, antecesora de la actual Facultad de Derecho de la Universidad de Costa Rica, de compartir la infraestructura (Consejo Universitario de la Universidad de Costa Rica, 1944).

Con respecto a su presupuesto, este no era ubicado en las partidas disponibles para los gastos de la Facultad de Ciencias Económicas y Sociales, como sí lo hacían para el resto de instancias académicas que la conformaban; incluso la dirección de la Escuela de Servicio Social no tenía incidencia política en las decisiones del decanato ni en el Consejo Universitario (Consejo Universitario de la Universidad de Costa Rica, 1944). Para ilustrar uno de esos aspectos señalados, la siguiente tabla permite ver el presupuesto de la Escuela de Servicio Social durante el periodo en mención.

De esta manera, como parte de la reestructuración de la Facultad de Ciencias Económicas y Sociales, a partir de marzo de 1947 la Escuela de Servicio Social dejó la figura de escuela anexa y pasó a llamarse Sección de Sociología y Servicio Social (Consejo Universitario de la Universidad de Costa Rica, 1947). Esto implicaba que en términos académicos y administrativos, la Escuela se vinculaba mayormente a la Facultad de Ciencias Económicas y Sociales de la Universidad de Costa Rica. 
A partir de lo planteado, se explicita como los requerimientos institucionales, en consonancia con las condiciones históricas de un proyecto reformista que se empezaba a gestar, mediaron en la demanda de profesiones como el Trabajo Social para que intervinieran en el plano operativo de la política social. De allí la premisa teórica antes señalada sobre la legitimidad de la profesión y su institucionalización, dado que ambas condiciones provienen del requerimiento institucional por la atención de las demandas sociales relacionadas con la llamada cuestión social, antes que del acervo de conocimientos que el espacio académico provee.

\section{TABLA 1}

Presupuesto de la Escuela de Servicio Social durante su periodo como escuela anexa de 1944 a 1947

\begin{tabular}{cc}
\hline AÑO & CURSO \\
\hline $1944-1945$ & $\phi 18000$ \\
$1945-1946$ & $\varnothing 18800$ \\
$1946-1947$ & $\varnothing 19400$ \\
\hline
\end{tabular}

Nota: Elaboración propia a partir de actas del Consejo Universitario de la Universidad de Costa Rica (1944; 1945; 1946).

No obstante, la explicación de la institucionalización de la formación profesional del Trabajo Social costarricense, no se agota en el ascenso del proyecto reformista; por el contrario, es un punto de referencia. Se plantea de esa forma, dado que las adscripciones políticas, ideológicas, teóricas y metodológicas que reciben a la Escuela, inicialmente, en la Facultad de Ciencias Económicas y Sociales de la Universidad de Costa Rica, así como de su cuerpo docente, median particularmente en el proceso de institucionalización de la formación profesional.

En el apartado siguiente, referente a los fundamentos de la formación en la Escuela de Servicio Social, se procederá a profundizar en el tema de la institucionalización, dado que es oportuno señalar la característica que existe en el Trabajo Social de que su legitimidad es provista, en mayor medida, por la institucionalidad.

En ese sentido, tal característica profesional, relacionada con la legitimidad que le provee la institucionalidad al Trabajo Social, permite entender por qué la Escuela de Servicio Social fue subsidiada a través del apoyo institucional, durante el periodo que tardaron sus gestiones para vincularse a la Universidad de Costa Rica; de igual forma, posibilita una explicación de las razones por las que una vez dentro como unidad académica, atravesó una serie de oscilaciones institucionales asociadas con los cambios experimentados por la Universidad, pero también por el papel subordinado que tuvo frente a otras instancias académicas. 
Ahora bien, junto con las particularidades señaladas, los frecuentes cambios en el currículo fueron otras de las circunstancias identificadas en la Escuela de Servicio Social para el periodo en estudio. Por ejemplo, en 1942 se impartieron cuatro cursos preparatorios previos a la puesta en marcha de un programa de cuatro años. Los mencionados cursos preparatorios fueron: 1) Elementos de Derecho del Trabajo y Seguro Social, 2) Elementos de Asistencia Social, 3) Elementos de Higiene Social y 4) Medicina Social (Archivo Nacional de Costa Rica, 1942). Originalmente, la propuesta de Beeche Luján consistía en que una vez ejecutados los cuatro cursos de preparación, fueran suprimidos para dar paso a un plan de estudios de cuatro años (Archivo Nacional de Costa Rica, 1942).

Con respecto a esa propuesta curricular cuatrienal, cabe mencionar que fue diseñada por la American Association of School of Social Work en 1932 (Angulo, 2014). De igual forma, la señora Alice Shaeffer, trabajadora social estadounidense graduada en la Escuela de Servicio Social de la Universidad de Chile, fue quien asesoró a Beeche Luján en la implementación inicial del currículo (Archivo Universitario Rafael Obregón Loría, 1954).

No obstante, tal propuesta curricular no logró concretarse. Según Angulo (2014), las razones se encuentran en que "las condiciones sociales, culturales y políticas de Costa Rica no coincidían con este proyecto, así como la influencia de las instituciones en la formación incidió en el establecimiento de otras discusiones" (p. 221).

En ese sentido, el Consejo Universitario aprobó para el año 1945 un plan de estudios mediante la llamada reforma de los tres años, dicho plan fue enviado a las autoridades universitarias para su validación en el año 1944 (Angulo, 2014). Este aspecto da pie para comprender las razones por las cuales los nombres de los cursos con los que el Consejo Universitario designó el nombramiento de los docentes durante el periodo 1944 a 1946, no corresponden con los nombres de los cursos originalmente propuestos en la malla curricular de 1942 (Consejo Universitario de la Universidad de Costa Rica, 1944; 1946).

Tal y como se mencionó, la escuela de instancia anexa pasó en 1947 a formar parte de una sección de la Facultad de Ciencias Económicas y Sociales, llamada Sección de Sociología y Servicio Social (Consejo Universitario de la Universidad de Costa Rica, 1950). Sin embargo, lo importante de señalar en este momento del análisis, es que dichas oscilaciones significaron, nuevamente, un cambio formal en el currículo de la Escuela de Servicio Social.

En ese sentido, en 1948 se planteó una nueva malla curricular con una duración de cinco años, en la cual se identifica la inclusión de las Ciencias Sociales en la formación profesional. En esa línea, en febrero de 1950 el Consejo Universitario aprobó una nueva reforma al plan de estudios (Consejo Universitario de la Universidad de Costa Rica, 1950).

La nueva reforma a la malla curricular la extendía a cinco años. Una de sus principales novedades era la conformación de un bloque común de cursos que compartía con el resto de las carreras que ofrecían las diferentes secciones de la 
Facultad de Ciencias Económicas y Sociales; posteriormente a ese nivel de un año de duración, se impartían los cursos con mayor atinencia al Trabajo Social (Consejo Universitario de la Universidad de Costa Rica, 1950).

No obstante, en diciembre de 1952 el currículo sufrió, nuevamente, modificaciones; en esta ocasión, mantenía el bloque común antes mencionado, pero centraba los cambios en algunos de los cursos específicos de Trabajo Social, de allí que esta nueva reforma prestó atención a los niveles posteriores al primer año de la carrera (Consejo Universitario de la Universidad de Costa Rica, 1952).

\section{LOS FUNDAMENTOS INICIALES DE LA FORMACIÓN PROFESIONAL EN LA ESCUELA DE SERVICIO SOCIAL}

La comprensión de los fundamentos que acompañaron la formación académica en la Escuela de Servicio Social para el periodo en estudio, puede ser tratada a partir de dos mediaciones que la condicionaron. Una de ellas son las condiciones históricas a través de las cuales el Estado, mediante la creciente política social, atendió la cuestión social y la otra es el movimiento que a lo interno de la categoría profesional adquirieron determinadas teorías en correspondencia con ciertas matrices filosóficas (Fallas, 2010).

El fundador y primer director de la Escuela, Héctor Beeche Luján, propuso la formación profesional con un carácter criminológico; sin embargo, la propuesta nunca logró concretarse. Esto se debió a que el abordaje de la cuestión social demandaba otros perfiles para su atención, sumado a que la presencia de diversos actores en la junta directiva de la Escuela, moldearon la malla curricular hacia otras direcciones (Fallas, 2010).

De esta forma, las instituciones públicas se convirtieron en elementos fundamentales en la influencia académica de la Escuela de Servicio Social, en tanto representaban expresiones concretas del abordaje estatal en el conflicto social, plano de actuación de la profesión (Angulo, 2014). Es comprensible entonces que las primeras propuestas de formación académica se centraran en preparar cuerpos técnicos con un adecuado adiestramiento, para intervenir en los requerimientos operativos de la naciente política social que ejecutaban las instituciones públicas (Angulo, 2014).

Es así como se encuentran reflexiones en la formación académica vinculadas con la salud e higiene, escenario de ampliación estatal para el periodo en estudio. De igual forma, se identifican contenidos en el área de la administración y la economía, dada la importancia que poseían tanto por las intervenciones de los organismos internacionales que incursionaban para el periodo en la institucionalidad pública, así como por el diálogo que experimentó la Escuela con las ciencias económicas, producto de su adscripción a la Facultad de Ciencias Económicas y Sociales a su llegada a la Universidad de Costa Rica (Fallas, 2010).

Por su parte, la Escuela profesionalmente se vinculó con corrientes asociadas al Trabajo Social norteamericano, conocido como Social Work. Dicha propuesta 
confluye en el Servicio Social de caso individual, el cual se sustentaba, en cierta medida, por las corrientes pragmáticas y empiristas (Fallas, 2010). Ante esas particularidades en la formación académica, la Escuela de Servicio Social inició en el año 1952 con la implementación de contenidos teóricos vinculados con la intervención en las localidades (Angulo, 2014).

A partir de lo expuesto, es válido indicar que se presentó una formación académica con influencia inicialmente de las expresiones de atención de la cuestión social en el plano institucional costarricense, así como del diálogo entablado por la Escuela con los saberes asociados a la Facultad de Ciencias Económicas y Sociales y su cuerpo docente. De igual forma, se evidenció una fuerte influencia del Trabajo Social estadounidense a través del Social Work y de la incursión en la escena institucional de los congresos y los organismos internacionales, tales como los congresos panamericanos, la Organización de las Naciones Unidas (ONU) y la Organización de los Estados Americanos (OEA) (Angulo, 2014; Fallas, 2010).

\section{LAS MEDIACIONES POLÍTICAS DE LA PROFESIÓN EXPRESADAS EN LOS PRIMEROS AÑOS DE FUNCIONAMIENTO DE LA ESCUELA DE SERVICIO SOCIAL}

Ahora bien, la estrecha consonancia con la institucionalidad costarricense que entrelazó, tendencialmente, la constitución profesional, permitió para el periodo en estudio identificar cómo en función de la fracción política al mando de la esfera estatal, así se estructuraba la Escuela de Servicio Social. Es decir, se vuelve explícita la mediación política que constituye a la profesión, la cual deviene de la estrecha relación del Trabajo Social con la institucionalidad estatal.

Al iniciar el análisis en esta línea, cabe señalar que la Escuela fue dirigida en 1942 por Beeche Luján y en algunos momentos no con igual permanencia por el Dr. Santi Quirós Navino (Valverde, 1992). Muestra de ello es cuando en 1945 el señor Quirós sustituyó a Beeche Luján en el momento en que este último pidió al Consejo Universitario un permiso sin goce de salario, debido a que presentaba problemas de salud (Consejo Universitario de la Universidad de Costa Rica, 1946).

Durante el año en que Beeche Luján buscó respaldo para la fundación de la Escuela de Servicio Social, apoyó sus intenciones en el beneplácito de algunas figuras de relevancia institucional. En la siguiente tabla se procede a exponer el nombre de las personas a las que acudió Beeche Luján durante su proceso de constitución de la Escuela de Servicio Social.

De igual forma, la siguiente tabla menciona los nombres propuestos por Beeche Luján para los cursos de nivelación de 1942, previo a la ejecución del plan de estudios de cuatro años.

Al respecto, es importante señalar que la planta docente, así como las personas en las cuales buscó respaldo Beeche Luján para la fundación de la Escuela de Servicio 
Social, se encontraba asociada al proyecto reformista relativamente amalgamado bajo el gobierno oficial de Calderón Guardia. En esa línea, es importante indicar que como parte de la reorganización que atravesó la Facultad de Ciencias Económicas y Sociales en 1947, el Consejo Universitario optó por nombrar una nueva planta docente.

\section{TABLA 2}

\begin{tabular}{cc}
$\begin{array}{l}\text { Nombres de las personas que apoyaron a Beeche Luján en su } \\
\text { pretensión de fundar una Escuela de Servicio Social en } 1942\end{array}$ \\
\hline NOMBRE & PROFESIÓN \\
\hline Guillermo Padilla & Médico \\
Mario Luján & Médico \\
Alfredo Sánchez & Médico \\
Antonio Bruck & Se desconoce \\
Abel Guier Alvarado & Psicólogo \\
Oscar Barahona Streber & Se desconoce \\
\hline
\end{tabular}

Nota: Elaboración propia a partir de la documentación identificada en el Archivo Nacional de Costa Rica (1942).

TABLA 3

Nombre de las personas propuestas por Beeche Luján para ejercer la docencia en los cursos transitorios de 1942

\begin{tabular}{ccc}
\hline DOCENTE & CURSO & PROFESIÓN \\
\hline Oscar Barahona & $\begin{array}{c}\text { Derecho del Trabajo y } \\
\text { Seguridad Social } \\
\text { Mario Luján }\end{array}$ & Abogado \\
Armando Guevara & Medicina Social & Médico \\
Antonio Bruck & Asistencia Social & Médico \\
\hline
\end{tabular}

Nota: Elaboración propia a partir de la documentación identificada en el Archivo Nacional de Costa Rica (1942).

En ese proceso, fue designado como director el médico José Armando Guevara (Angulo, 2014); no obstante, el sacerdote Francisco Herrera Mora fue nombrado en el cargo de director durante el cierre de sus estudios universitarios en 
Estados Unidos en el año 1947 (Valverde, 1992). De esa forma, en concordancia con los mencionados cambios que experimentó la Escuela, significativamente, también se renovó la planta docente. En cuanto a ello, se procede a exponer en la siguiente tabla los nombres de los docentes que para tales efectos fueron nombrados por el Consejo Universitario.

Tal y como se ha venido planteando, la mayoría de docentes presentes en este cartel se encontraban en estrecha consonancia con el proyecto reformista que, posteriormente al conflicto armado de 1948, fue asediado por el bando vencedor. La Universidad de Costa Rica empezó a forjar a lo interno una intelectualidad asociada al proyecto socialdemócrata, que después de la coyuntura histórica mencionada, expulsó a docentes de la Universidad contrarios a esa línea política emergente (Fumero, 1997). De modo que los profesores de la Escuela de Servicio Social fueron blancos de tales consecuencias.

\section{TABLA 4}

Nombre de los docentes designados según curso en 1947, como parte de la reestructuración de la Escuela de Servicio Social durante su tránsito de escuela anexa a sección de la Facultad de Ciencias Económicas y Sociales

\begin{tabular}{|c|c|c|}
\hline DOCENTE & CURSO & PROFESIÓN \\
\hline Viriato Camacho & Psicología & Educador \\
\hline Fabián Dobles & Bienestar de la Familia y el Niño & Abogado \\
\hline Armando Guevara & Medicina Social & Médico \\
\hline Héctor Beeche Luján & Ciencia Penitenciaria & Abogado \\
\hline José Guerrero & Estadística Biodemográfica & Se desconoce \\
\hline Santi Quirós & Patología Social & Médico \\
\hline Francisco Herrera & Servicio Social & Trabajador Social \\
\hline Benjamín Núñez & Investigaciones Sociales & Sociólogo \\
\hline Mariano Coronado & Higiene Mental & Médico \\
\hline Roberto Campabadal & Higiene Social & Farmacéutico \\
\hline Fernando Jones & Servicio Social & Se desconoce \\
\hline
\end{tabular}

Notas: Elaboración propia a partir de las actas del Consejo Universitario de la Universidad de Costa Rica (1947). 
Tomando en cuenta la mediación política que subyace en la profesión, es comprensible por qué una Escuela como la de Servicio Social sufrió en mayor medida la reconfiguración política de la guerra civil costarricense de 1948: al estrechar sus cimientos profesionales en el plano institucional, su adscripción acrítica a la esfera estatal le hacía maleable al proyecto político de turno.

Es decir, para el periodo en estudio, al cambiar la fracción del reformismo en la conducción estatal, cambiaba en igual medida la estructura académica de la Escuela de Servicio Social. Cobran sentido, nuevamente, los planteamientos anteriormente mencionados respecto a que la profesión obtiene mayor legitimidad por la vía institucional, antes que del sistema de saberes que la formación académica le concede.

En ese orden de ideas, es comprensible que durante sus primeros seis años (1942-1948), los representantes del proyecto político al mando del Estado fueran los responsables de dar el beneplácito para la fundación de la Escuela, hasta incluso ejercer ellos la docencia. A partir de lo expuesto, se comprende que al cambiar el proyecto político de fracción, después de la guerra civil costarricense de 1948, la institucionalidad se alineara a esa propuesta $\mathrm{y}$, consecuentemente, la unidad académica al tener una estrecha relación con la esfera estatal, sufriera el impacto de tal reconversión.

Ante la inflexión institucional acarreada por el violento conflicto armado de 1948, los docentes de la Escuela de Servicio Social denunciados y a quienes el Consejo Universitario les pidió que dimitieran de sus puestos fueron, tal como se indicó, los relacionados con el proyecto político en declive. De la tabla 4, las personas expulsadas que ejercieron la docencia en la Escuela de Servicio Social fueron: Roberto Campabadal, Fabián Dobles, José Armando Guevara y el propio fundador de la Escuela, Héctor Beeche Luján (Fumero, 1997).

En adelante, las oscilaciones de la Escuela de Servicio Social transitaron en mayor medida a partir del proyecto en ascenso institucional: el socialdemócrata; el cual medió en términos políticos, ideológicos, teóricos y metodológicos las siguientes dos décadas de la Escuela de Servicio Social (Angulo, 2014; Fallas, 2010).

\section{A MANERA DE CIERRE}

En el artículo se evidenció cómo el ascenso de la política reformista costarricense de la década de 1940, principalmente las acciones relacionadas a la política social, propició las condiciones históricas para la constitución del Trabajo Social, demandando instancias para su formación académica. De esta manera, la institucionalización de la formación a través de la Escuela de Servicio Social tiene como escenario fundacional determinadas condiciones históricas (económicas, políticas, sociales y culturales) que le permitieron su constitución.

Debido a la legitimidad institucional que caracterizó a la profesión antes que el acervo de saberes teóricos que un escenario académico pudo ofrecerle, la 
incursión de la Escuela de Servicio Social en la Universidad de Costa Rica se encontró determinada por una serie de oscilaciones administrativas y curriculares.

Por su parte, en cuanto a los fundamentos de la formación académica en la Escuela de Servicio Social, se logra identificar que se encontró mediada por las características de las estrategias estatales para abordar la cuestión social, así como por la reproducción a lo interno de la profesión de determinadas tradiciones teóricas.

Igualmente, se mostró que la estrecha vinculación entre la legitimidad profesional y las demandas estatales, hizo que la Escuela de Servicio Social fuera maleable a las propuestas de la fracción reformista al mando de la institucionalidad. Sobre ese aspecto, se argumentó que conforme ascendía a la estructura estatal una determinada fracción del proyecto político reformista, el andamiaje de la unidad académica oscilaba ante tal inflexión.

Es así como la investigación destaca que la institucionalización de la formación del Servicio Social fue una exigencia de las condiciones históricas orientadas por el creciente reformismo de la década de 1940, mediada por las características institucionales para la atención del conflicto social, así como por las particularidades que afrontó la primera unidad académica tanto por su personal docente y administrativo, como por las repercusiones de formar parte de la Facultad de Ciencias Económicas y Sociales, al momento de ingresar a la Universidad de Costa Rica.

No obstante, al deberse la profesión constitutivamente a tales características de naturaleza política, como es el caso de la política social, y consecuentemente al Estado, al menos para el periodo de estudio, la Escuela de Servicio Social se encontró condicionada, principalmente, por las tensiones que se presentaban en la institucionalidad pública costarricense.

\section{NOTAS}

1 Se debe señalar que en sus inicios el Trabajo Social era denominado Servicio Social; por lo tanto, la Escuela era llamada para el periodo en estudio Escuela de Servicio Social.

\section{REFERENCIAS}

Angulo, S. (2014). Fundamentos de la complejidad agraria-campesina y rural en la formación académica de la Escuela de Servicio Social de la Universidad de Costa Rica, 1940-1970. (Tesis de Maestría en Trabajo Social). Universidad de Costa Rica, Costa Rica.

Archivo Nacional de Costa Rica. (1942). Fondo Gobernación, sub-fondo Educación. (Signatura 4722, ff. 60-78). Costa Rica: Archivo Nacional de Costa Rica.

Archivo Universitario Rafael Obregón Loría. (1954). Sub-Fondo Facultad de Ciencias Económicas y Sociales. (Caja 10455). Costa Rica: Archivo Universitario Rafael Obregón Loría. 
Consejo Universitario de la Universidad de Costa Rica. (10 de marzo de 1942). Actas históricas del Consejo Universitario. (Acta 004). Recuperado de http://www.cu.ucr.ac.cr/uploads/tx_ucruniversitycouncildatabases/minute/1942/1942-004.pdf/

Consejo Universitario de la Universidad de Costa Rica. (7 de mayo de 1944). Actas históricas del Consejo Universitario. (Acta 002). Recuperado de http://www.cu.ucr.ac.cr/actas/1944/1944-002. pdf/

Consejo Universitario de la Universidad de Costa Rica. (18 de julio de 1944). Actas históricas del Consejo Universitario. (Sesión extraordinaria E08). Recuperado de http://www.cu.ucr.ac.cr/ actas/1944/1944-E08.pdf/

Consejo Universitario de la Universidad de Costa Rica. (10 de setiembre de 1946). Actas históricas del Consejo Universitario. (Sesión No. 037). Recuperado de http://www.cu.ucr.ac.cr/actas/1946/1946-037.pdf/

Consejo Universitario de la Universidad de Costa Rica. (18 de febrero de 1947). Actas históricas del Consejo Universitario. (Sección extraordinaria E3). Recuperado de http://www.cu.ucr.ac.cr/actas/1947/1947-E03.pdf/

Consejo Universitario de la Universidad de Costa Rica. (16 de febrero de 1950). Actas históricas del Consejo Universitario. (Sesión extraordinaria E06). Recuperado de http://www.cu.ucr.ac.cr/actas/1950/1950-E06.pdf/

Consejo Universitario de la Universidad de Costa Rica. (1 de diciembre de 1952). Actas históricas del Consejo Universitario. (Sesión 51). Recuperado de http://www.cu.ucr.ac.cr/uploads/tx_ucruniversitycouncildatabases/minute/1952/51.pdf/

Fallas, Y. (2010). Fundamentos teórico metodológicos del Trabajo Social costarricense, 1942/1970. (Tesis de Maestría en Trabajo Social). Universidad de Costa Rica, Costa Rica.

Fumero, P. (1997). Se trata de una dictadura sui generis. Anuario de Estudios Centroamericanos, 23(12), pp. 115-142.

Montaño, C. (2000). La naturaleza del servicio social: un ensayo sobre su génesis, su especificidad y su reproducción. Brasil: Editorial Cortez.

Netto, J. (1992) Capitalismo Monopolista y Servicio Social. Brasil: Editora Cortez.

Valverde,L. (1992) Los inicios del Trabajo Social en Costa Rica: El padre Herrera. Revista de Ciencias Sociales. V. (N. 56), pp. 43-50. 